\title{
Hablar de Dios, en América Latina, desde los socialmente insignificantes
}

\author{
Gustavo Gutiérrez, \\ Lima, Perú.
}

Estamos aquí, una vez más, para "acompañar a Monseñor". Es una frase que escuché, hace veinticinco años, de labios de los que hacían largas colas para rezar junto a su tumba, en la catedral. Por lo menos cuarenta personas murieron por esa razón, el día del entierro. Pero nos sabemos, también, acompañados por ese gran testigo de la resurrección que, como Jesús, dio su vida por fidelidad y amor. Acompañados y, en cierto modo, interpelados por quien nos recordó que la vida, y no la muerte, es la última palabra de la existencia y la historia humanas. Estamos acá para pedirle al Dios de la vida, por medio de san Romero de América Latina - como dice nuestro querido obispo-poeta, Pedro Casaldáliga, y como lo venera el Pueblo de Dios - que nos ayude en nuestro caminar, en estas tierras de penurias y esperanzas.

Los organizadores de este encuentro - a quienes agradezco la invitaciónme han propuesto que trate el tema: "Hablar de Dios, en América Latina, desde los socialmente insignificantes". Mons. Romero afirmaba "Cristo es la homilía de Dios". Podemos decir que él hizo de su vida, pasión y muerte una homilía (palabra compartida) de Jesús. Nos mostró que la palabra auténtica no es llevada por el viento, sino que ella "queda", como decía Monseñor. Una palabra que no cesó de recordar lo que los pobres, los insignificantes, aquellos que viven una situación de muerte prematura e injusta, representan para hablar del Dios de la vida. La teología es, precisamente, un hablar, un lenguaje acerca de Dios. Todo un mundo social, cultural y psicológico interviene en la elaboración del discurso sobre la fe.

Cuando se atraviesan situaciones humanas extremas, de sufrimiento, de postergación y de injusticia, como en América Latina y el Caribe - como en El Salvador-, las preguntas calan hondo y conducen al corazón de las cosas; al mismo tiempo, el hablar teológico se hace respetuoso de la diversidad de las 
condiciones sociales, culturales y religiosas de personas y pueblos. En efecto, si no vamos al mundo del dolor cotidiano, de la vivencia de ver violados los derechos más elementales, de la angustia que consume, pero, asimismo, si no

- tenemos en cuenta las hondas experiencias de alegrías sencillas, de la esperanza que - pese a todo- se enciende permanentemente, en medio de situaciones inhumanas, el quehacer teológico no adquiere espesor. Y fácilmente puede contaminarse de un cierto burocratismo y de una voluntad de poder contrarios al espíritu evangélico.

Nos proponemos, en estas páginas, examinar algunos rasgos que caracterizan el lenguaje sobre Dios, que nace en el mundo de la insignificancia social. Examinaremos, en primer lugar, lo que entendemos por el reto que la pobreza, en especial la pobreza extrema, que algunos prefieren llamar miseria, plantea al hablar de Dios; luego veremos el sentido y la ubicación que, en función de lo anterior, damos a la perspectiva de la preferencia por el pobre. Finalmente, tocaremos la cuestión de la unidad y la diversidad de los lenguajes sobre Dios.

\section{Una realidad compleja}

La pobreza es una realidad polifacética, inhumana e injusta; consecuencia, sobre todo, de la forma como se piensa y se organiza la vida en sociedad. La pobreza es un hecho complejo. No se limita, por lo tanto, sin que esto signifique negar su importancia, a la vertiente económica. La realidad de países multirraciales y multiculturales, como lo son una buena parte de los latinoamericanos, el Perú entre ellos, nos puso rápida y directamente ante esa diversidad ${ }^{1}$. Visión reforzada por la compleja comprensión que la Escritura, en ambos testamentos, tiene de los pobres: aquellos que mendigan para vivir, las ovejas sin pastor, los ignorantes de la ley, aquellos que son llamados "los malditos", en el evangelio de Juan $(7,49)$, las mujeres, los niños, los extranjeros, los pecadores públicos y los enfermos de males graves.

Presente desde un inicio, como problema y como enfoque, esta complejidad fue ahondada por la reflexión teológica latinoamericana, siguiendo variadas líneas, en los años siguientes. Precisamente, esa percepción nos llevó a las tempranas expresiones de "no persona" y de "insignificante" para referimos a los pobres ${ }^{2}$. Con

1. En la reflexión teológica latinoamericana la afirmación de la complejidad de la pobreza viene - decíamos- de considerar sus "coordenadas económicas, sociales, culturales, raciales"; por ello, el pobre es visto como miembro de "pueblos, razas y clases sociales" (Teología de la liberación, Lima, 1971, pp. 251 y 226, cfr. también pp. 255, 354) y de "las clases populares explotadas, las culturas oprimidas, las razas discriminadas" ("Praxis de liberación y fe cristiana", en Signos de liberación (Lima, 1973), a lo cual se añade que "la mujer de esos sectores sociales es doblemente explotada, marginada y despreciada" (Teología desde el reverso de la historia, Lima, 1977, p. 34, nota 36).

2. Con la expresión no persona buscábamos designar al interlocutor de las teologías de la liberación, en contraste con el no creyente, interlocutor de las teologías modernas. La expresión de insignificante apuntaba a su falta de peso en la sociedad. 
ellas se quería subrayar lo que tienen en común todos los pobres: la ausencia del reconocimiento de su dignidad humana y de su condición de hijas e hijos de Dios, sea tanto por razones económicas, como raciales, de género, culturales, religiosas u otras. Condiciones humanas, estas últimas, que la mentalidad dominante de nuestras sociedades no valora, creando una situación desigual e injusta.

La pobreza no es una fatalidad, sino una condición; no es un infortunio, sino una injusticia. Es resultado de estructuras sociales y de categorías mentales y culturales. Está ligada al modo cómo se ha construido la sociedad, en sus diversas manifestaciones. Es fruto de manos humanas: estructuras económicas y atavismos sociales, prejuicios raciales, culturales, de género y religiosos, acumulados a lo largo de la historia, intereses económicos cada vez más ambiciosos ${ }^{3}$; por lo tanto, su abolición se halla también en nuestras manos.

Actualmente, disponemos de los instrumentos - sujetos al examen crítico de rigor- que permiten conocer mejor los mecanismos económico-sociales y las categorías en juego. Analizar esas causas es una exigencia de honestidad, y, a decir verdad, es el camino obligado, si queremos, en realidad, superar un estado de cosas injusto e inhumano. Punto de vista que - sin olvidar que en la pobreza de los pueblos intervienen variados factores - desvela el papel que tiene la responsabilidad colectiva en este asunto $y$, en primer lugar, la de quienes tienen mayor poder en la sociedad.

Pese a la evidencia del asunto, no puede decirse, sin embargo, que esta perspectiva estructural se haya convertido en una opinión generalizada, en el mundo de hoy, ni tampoco en ambientes cristianos. Hablar de causas de la pobreza hace ver la delicadeza y, en verdad, la conflictividad de esta cuestión, razón por la cual muchos buscan soslayarlas. Uno de ellos es la dimensión planetaria de la situación de la gran mayoría de la población mundial. Esto vale para el conjunto de lo que entendemos por pobreza, aunque muchas veces los estudios insistan, más bien, en su vertiente económica, sin duda, la más fácil de medir. Por largo tiempo, las personas sólo conocieron la pobreza que tenían cerca, en su ciudad o, a lo sumo, en su país. Esto cambió, cualitativamente, con la facilidad de información, que se fue adquiriendo; así aquello que antes era distante y remoto, se ha hecho próximo y cotidiano. Además, los datos y los estudios sobre la pobreza masiva, realizados por un sinnúmero de organizaciones, en nuestros días, se multiplican, y perfilan sus métodos de investigación. No pueden, pues, ser ignorados.

Otro rasgo que ha modificado, asimismo, nuestra aproximación a la pobreza es su profundización y el aumento de la brecha entre las naciones y personas

3. En ocasiones, esas posturas se manifiestan de manera teórica y articulada, como es el caso, en Estados Unidos, del libro de S. Huntington (autor del cuestionable The Clash of Civilizations) sobre la inmigración latinoamericana a Estados Unidos: Who Are $E e$ ? New York-London, 2004. 
más ricas y las más pobres. Esto, a juicio de ciertos economistas, está llevando a lo que se ha calificado de neodualismo: la población mundial se coloca cada vez más en los dos extremos del espectro económico y social. Una de las líneas divisorias es el conocimiento científico y técnico, constituido en el eje más importante de acumulación de la actividad económica y cuyos avances han acelerado la ya desenfrenada explotación - y depredación - de los recursos naturales del planeta, los cuales son un patrimonio común de la humanidad. Estos factores han acrecentado la distancia que anotábamos.

No obstante, el asunto no se limita al aspecto económico de la pobreza y la insignificancia. En el espacio creado por esa disparidad creciente, intervienen y se entrecruzan los elementos mencionados antes: los que vienen del terreno económico, por un lado, con los referentes a las cuestiones de orden cultural, racial, y de género, por el otro lado. Esto último ha llevado a hablar, con razón, de una feminización de la pobreza. Las mujeres constituyen, en efecto, el sector más afectado por la pobreza y la discriminación, sobre todo si pertenecen a culturas o a etnias postergadas. Si bien, la cuestión ha alcanzado ahora proporciones escandalosas ${ }^{4}$, el proceso de acentuación de esa distancia estaba en marcha, desde hace décadas, lo cual explica la alarma que ya provocaba entonces.

Hoy - y este hoy lleva ya un buen tiempo-, la inhumanidad e injusticia de la pobreza, la ignorancia de sus causas y la percepción de su complejidad, extensión, y hondura, tengamos o no una experiencia directa de ella, no pueden ser disculpadas.

\section{El reto de la pobreza y el anuncio del evangelio}

La pobreza es una realidad inhumana, injusta y, a la vez, susceptible de eliminación. Toda tolerancia, acomodo teórico, actitud ambigua o uso espiritualista de ella es un insulto a las personas concretas que la padecen. Es más, la pobreza, la condición de insignificancia humana y de muerte temprana e injusta, es una condición opuesta a la voluntad de vida y amor del Dios de la Biblia

Se trata de un serio desafío al modo de entender la fe y de dar testimonio de ella. Los términos "no persona" e "insignificante" no sólo expresan -como lo decíamos líneas antes - el denominador común de los diferentes aspectos de la pobreza, sino que nos indican también la hondura de la injusticia y la gravedad de esa condición. La insignificancia social y humana no es un asunto que se enfrenta sólo colocándolo en el casillero de los problemas económicos y sociales; va mucho más allá. La envergadura de su globalidad y complejidad nos invita a releer el mensaje cristiano.

4. Los informes anuales del PNUD y del Banco Mundial, entre otros, lo comprueban año con año. 
Subrayar el carácter teológico de las preguntas que acarrea la pobreza no significa, de ningún modo, olvidar que ella y la injusticia social que la provoca tienen una inevitable y constitutiva dimensión socio-económica y cultural. Esto es evidente. Pero no se confina a lo que alguna vez, se llamó "la cuestión social". La pobreza y la opresión, con su carga de muerte injusta y prematura, lanzan un cuestionamiento radical y global a la conciencia humana, y a la manera de vivir y aproximarse a la fe cristiana, que ve en el rechazo al amor al otro, el pecado, la raíz última de la pobreza y la deshumanización ${ }^{5}$. Sin embargo, no hay que olvidar que los grandes retos que interpelan a la fe cristiana proporcionan, a la vez, importantes elementos para configurar un campo hermenéutico, que nos conduce a una relectura del mensaje bíblico y a discernir el camino por emprender como discípulos de Jesús. Es algo que antes ha sucedido muchas veces, recordarlo permite entender el sentido de una teología como la de la liberación, que busca tomar en serio el desafío de la pobreza, en el hoy de nuestra historia. Para decirlo brevemente: la condición de los pobres interroga el corazón del mensaje cristiano y suministra perspectivas para responder con creatividad ${ }^{6}$. En ese horizonte se ubica lo que llamamos la opción preferencial por el pobre.

Las raíces de esa formulación - nacida en los años que precedieron a Puebla - están en el inicio de una reflexión teológica, hacia 1967. Constituyen la respuesta a la interpelación que viene de la pobreza. A esas fuentes hay que remitirse para captar su significado. En la reflexión aludida se formulan tres acepciones de la pobreza, que Medellín hizo suyas, dándoles un apoyo decisivo. Posteriormente, se recogen esas distinciones, con el dinamismo propio de una frase, por medio de tres palabras: pobre, preferencia y opción. Asumida en Puebla, tendrá una resonancia de la cual habría carecido, si hubiese permanecido exclusivamente en el campo de las publicaciones de orden teológico y en ciertos círculos eclesiales.

Un punto central de la elaboración teológica en que se sustenta esa opción, algo así como el piso sobre el cual se construye, fue afirmar de una forma descarnada que la pobreza real, vivida por los tenidos como insignificantes por el "otro" de los sectores dominantes de esta sociedad, es una situación inhumana, rechazada por el Dios de la Biblia. Por ello, en un análisis de fe, debe ser calificada como un mal, aserción que vale cualesquiera que sean las formas que esa condición

5. Con razón, Ignacio Ellacuría anotaba, en 1983: "El concepto teológico del pobre ha vuelto a cobrar en los últimos quince años la relevancia excepcional que tuvo en la predicación de los profetas, en la evangelización de Jesús y en los momentos mejores de la Iglesia" ("Pobres", en C. Floristán y J. J. Tamayo, Conceptos fundamentales de pastoral, Madrid, 1983, p. 786.

6. El enfoque de la opción por el pobre está asociado a la consideración de los pobres no sólo como destinatarios de la evangelización, sino también como portadores de la buena nueva. Punto recogido en Puebla como "el potencial evangeliador de los pobres" (1147). 
de insignificancia e injusticia pueda adoptar. Se descarta, de este modo, toda idealización de la pobreza real y se hacen deslindes, que permiten comprender el sentido de lo que el evangelio llama la pobreza espiritual, así como del compromiso con los pobres.

Veamos cómo se presentan las cosas. Para simplificar, estableceremos un paralelo entre Medellín y Puebla, que recogen la elaboración teológica citada.

a) La pobreza real, o material, es un estado escandaloso e injusto, al que nos acabamos de referir, atenta contra la dignidad humana y es contrario a la voluntad de vida de Dios. Ella es "en cuanto tal, un mal" y un "fruto de la injusticia y el pecado" (Medellín, Pobreza, 4). Es la situación de los pobres por quienes se debe optar, según Puebla.

b) La pobreza espiritual es, en primer lugar, un sinónimo de infancia espiritual, uno de los temas más profundos del mensaje bíblico. Es "la actitud de apertura a Dios, la disponibilidad de quien todo lo espera del Señor" (ibíd. 4b). La viven aquellos, como los llamados "pobres de Yahvé", que aceptan el designio amoroso de Dios sobre sus vidas. El desprendimiento de los bienes materiales deriva de esa posición de fondo. Con ella se relaciona el término preferencia ${ }^{7}$, en la medida en que el pobre espiritual hace suyo el amor de justicia y gratuidad de Dios (cfr. Mt 6, 33), dirigido a todos, pero que se encuentra prioritariamente en relación con los pobres ${ }^{8}$.

c) El término opción proviene de la pobreza vista "como un compromiso" (ibíd. $4 c)^{9}$. Ahora bien, en la reflexión teológica aludida, el compromiso tiene una doble faceta: solidaridad con los pobres y protesta contra la

7. El término preferencia está ya en Medellín. En el documento Pobreza de la Iglesia hay un párrafo que liga la preferencia al compromiso con los pobres, que se llama justamente, "Preferencia y solidaridad".

8. La idea se halla en los años que van de Medellín a Puebla de diversas maneras. A título de ejemplo, he aquí algunas referencias, de las muchas de esos años. En el discurso de clausura de la conferencia de Medellín, el cardenal Landázuri, uno de los presidentes de la asamblea, reafirma: "nuestras prioridades son los pobres de este continente, es decir Cristo en ellos" (Signos de Renovación, Lima, 1969, p. 252). En textos inmediatamente posteriores a Medellín encontramos - provenientes de diferentes esquinas del continente - asertos como "Con preferencia por los más necesitados" ("Convención de presbíteros", Ecuador, 1970, en SL 143b), "nos solidarizamos con la condición de los oprimidos y les damos preferencia en nuestro ministerio" ("Declaración de la Asociación de sacerdotes", Puerto Rico, 1972, en SL 225); "compromiso prioritario con los pobres y marginados de nuestra tierra" ("El presbiterio de Asunción", Paraguay, 1972, en SL 74b). En los textos citados, el subrayado es nuestro.

9. Compromiso, precisamos, que no concierne sólo a los no pobres, los pobres mismos -importa decirlo- están llamados a hacerlo suyo. 
pobreza, en tanto situación inhumana. Se trata - dice, por eso, Medellín - de hacer nuestra la condición del pobre "para dar testimonio contra el mal que ella [la pobreza] representa" (ibíd.) ${ }^{10}$. Esta doble dimensión del compromiso (solidaridad y rechazo) es capital para comprender el sentido que debe darse al término opción.

\section{Un eje de vida y de reflexión}

La opción preferencial por el pobre nos recuerda un eje fundamental de la vida cristiana. Ella se despliega en tres niveles: el anuncio y testimonio del reinado de Dios, presente ya en la historia humana y llamado a transformarla; el de inteligencia de la fe, porque nos revela aspectos esenciales del Dios de nuestra fe y proporciona una perspectiva para el trabajo teológico y el caminar tras los pasos de Jesús, lo que conocemos como espiritualidad, en el nivel más profundo y sobre el cual todo lo demás reposa. Esa triple dimensión da vigor y alcance a la perspectiva que nos hace presente la opción por el pobre. En estas páginas, como lo hemos anotado, el acento está puesto en la segunda vertiente, en la teológica; incomprensible, claro está, si la desprendemos de las otras dos, a cuyo servicio se encuentra.

El enfoque de la opción por el pobre no es, no puede ser, evidentemente, algo exclusivo de una determinada teología. La exigencia y el significado del gesto hacia el pobre, en tanto consecuencia de la acogida del don del reinado de Dios, forman parte esencial del mensaje cristiano y, por consiguiente, deben estar, de un modo u otro, presentes en todo hablar sobre el Dios de Jesucristo. En tiempos recientes, un texto inspirador de la fórmula que comentamos, fue la propuesta de Juan XXIII al Concilio Vaticano acerca de "la iglesia [que] es y quiere ser la iglesia de todos y, especialmente, la iglesia de los pobres".

La expresión opción preferencial por el pobre es reciente como fórmula, pero, en cuanto al contenido, no es sino un recuerdo que llama a vivir, en nuestro tiempo, un dato capital de la revelación bíblica: la iniciativa del amor de Dios, "Dios nos amo primero" (1Jn 4,19).

Si la preferencia va a los pobres es, precisamente, porque se hallan en una situación injusta, contraria a la voluntad de vida de Dios y, por ello, inaceptable para un creyente. La preferencia es una denuncia de las desigualdades y marginaciones existentes, en la sociedad; busca, por ello, hacer que la afirmación de la universalidad del amor de Dios no se convierta en un manto piadoso, que oculte la inequidad social.

10. El texto de Puebla recupera los términos originales de esa reflexión, cuando precisa que estamos ante "la exigencia evangélica de la pobreza como solidaridad con el pobre y como rechazo de la situación en que vive la mayoría del continente" (1156). 
La teología que gira alrededor de esta opción es un discurso sobre la fe, que permite una relectura de las condiciones que se viven, en el mundo de hoy, con toda la novedad que ellas nos revelan, y no son pocas, de algo que - con insistencias, pero también con graves paréntesis - encontró, pese a todo, un lugar a lo largo del caminar histórico del pueblo de Dios. Nos referimos al lugar que ocupan los últimos de la historia, en el proyecto de liberación y humanización del reinado de Dios. Es relevante subrayarlo, no para disminuir la presente aportación de la vivencia cristiana y de la reflexión teológica, en una onda liberadora, que ha ligado su destino al sentido bíblico de la solidaridad con el pobre, sino para dibujar debidamente el ámbito en que ella se da, en tanto continuidad y ruptura con teologías anteriores. Y, sobre todo, con la experiencia cristiana y los senderos cotidianos, tomados para dar testimonio del reino, expresión del amor gratuito del Dios de Jesucristo, presente ya en la historia, pero todavía no plenamente, según la expresión clásica.

La fórmula programática de la "opción preferencial por el pobre" manifiesta lo más sustantivo - porque proviene del núcleo del mensaje cristiano- del aporte de la vida de la iglesia en Latinoamérica y el Caribe, y de la teología de la liberación, a la Iglesia universal. Las preguntas que a veces se plantean acerca del futuro de esta reflexión teológica deben tener en mente su relación factual y contemporánea con todo lo que dicha postura significa, vale decir sobre su presencia hoy, en la conciencia eclesial, e, incluso, más allá de ella. No es posible, por ello, separar, sin más trámite, esa opción de la perspectiva pastoral y teológica que - con los logros y las dificultades que conocemos - la ha puesto sobre el tapete en nuestros días. Si se habla de la opción por el pobre, se apunta al corazón de esa teología.

Pero, no olvidemos que la expresión no se refiere sólo a una cuestión de estrategia pastoral, por importante que ella pueda ser, para algunas decisiones concretas. Se trata de una cuestión más vasta y de mayor envergadura que va a lo medular de la vida cristiana y afecta diferentes vertientes de la existencia cristiana. La noción de preferencia hay que comprenderla en relación con la de universalidad del amor de Dios. Esto hace que esté íntimamente ligada a la exigencia de la solidaridad con los pobres y oprimidos. Sólo de este modo aparece su relevancia y puede responderse a riesgos que preocupan a algunos. Observaciones respetables que es importante tener en cuenta y que dan la ocasión de volver sobre puntos importantes

Preferencia y universalidad se implican de forma recíproca. Preferencia apunta a impedir que nos encerremos en una visión angosta y, finalmente, poco fecunda de la solidaridad con los últimos de la historia. Traer a la memoria esa prioridad, contribuye a darle a la opción por el pobre su radicalidad y mordiente. Sus fuentes son bíblicas, en eso consiste la verdadera radicalidad, remiten al horizonte del amor de Dios, universal y preferente a la vez. Por un lado, la universalidad sitúa el 
privilegio de los pobres en una ancha ruta y le exige rebasar continuamente sus eventuales límites; a su vez, la preferencia por los pobres da concreción y alcance histórico a dicha universalidad y le advierte del peligro de permanecer en un nivel abstracto y nebuloso ${ }^{11}$.

El término preferencia no intenta, de ningún modo, amortiguar la firme demanda de compromiso y solidaridad con el pobre. Se apeló a él en la experiencia y la reflexión de la Iglesia latinoamericana, en los años anteriores a Puebla, en continuidad con Juan XXIII y Medellín, y, sobre todo, respondiendo a sus raíces bíblicas. No se introdujo de forma subrepticia para recortar el alcance de la opción por el pobre, ni llegó a última hora. $\mathrm{Si}$, desde el comienzo de sus trabajos, Puebla llamó "Opción preferencial por los pobres" a uno de sus documentos, es porque - hemos recordado su recorrido - así se había comenzado a hablar, con las mismas o semejantes palabras, en el seno de muchas comunidades cristianas, documentos locales y escritos teológicos, en el tiempo que precedió a dicha conferencia $^{12}$. De hecho, el texto de ese documento es neto en afirmar la necesidad y la urgencia, y sin medias tintas, del compromiso con los oprimidos y marginados. La práctica de muchos en la Iglesia latinoamericana y caribeña había ya tomado ese rumbo desde Medellín, y su caminar fue refrendado en Puebla, de modo significativo. La preferencia, en la frase que analizamos, apela a la necesidad de la solidaridad con el pobre y a la recusación de la pobreza y sus causas.

Justamente, por esa razón, hay quienes, desde una posición ubicada al extremo opuesto del espectro, manifiestan, más bien, su preocupación por la indebida reducción a la que llevaría el término preferencia: se olvidaría - piensan - la afirmación fundamental de la universalidad del amor de Dios. En este caso, se teme que esa manera de hablar perfile una actitud que, limitando el alcance del amor cristiano, se dirija sólo a un sector de la humanidad, por numeroso que sea, descartando, como consecuencia, a otras personas de nuestra solicitud. Consideran, por ello, que se debe precisar que esa preferencia no es exclusiva.

A decir verdad, es un temor infundado, en cuanto al texto mismo (quizá no en cuanto a interpretaciones equivocadas que puedan darse de él), puesto que el mismo vocablo "pre-ferencia" nos habla ya - asumiendo una idea bíblica central - de solicitud prioritaria, de algo que es primero, no único; de predilec-

11. "Universalidad que para ser concreta debe pasar por la particularidad, por la toma de posición por aquellos que sufren injusticia" (G. Gutiérrez "La fuerza histórica de los pobres", en Signos de lucha y esperanza, Lima1978, p. XLI).

12. Este proceso está reflejado en el "Documento de trabajo" que trae, precisamente, un párrafo titulado "Opción preferencial por el pobre", recordando que la Iglesia reafirma esa opción ( $c f r$. 614, las fuentes señaladas son los aportes llegados de Bolivia, Perú, Venezuela, Ecuador México y Guatemala, en Puebla, Madrid, 1982, pp. 300-301). 
ción y de ningún modo de exclusión ${ }^{13}$. Son ciertas interpretaciones de la expresión y no la frase misma, las que han dado lugar a la advertencia de que estaríamos ante una opción con rasgos de exclusividad. En todo caso, no se trata ni de lo uno ni de lo otro: la preferencia ni merma la radical exigencia de solidaridad y justicia de la opción por los pobres, ni descarta a los que no pertenecen a ese estrato social. No estamos ante una preferencia que se puede, indiferentemente, tomar o dejar; si así fuese, se justificaría la desconfianza a que habíamos aludido, en primer lugar. Pero tampoco ante una preferencia que olvida el amor universal de Dios, como pretende el otro punto de vista. Ninguna de esas dos posiciones da cuenta cabal, nos parece, de la historia y de la significación de la fórmula en cuestión ${ }^{14}$.

Por otro lado, evitemos sobreestimar los términos que usamos en estas materias. Ellos indican una dirección y eso es lo importante. Referirse a la preferencia de Dios, en el contexto de su amor por toda persona, no puede hacerse sin emplear un lenguaje antropomórfico - $-i$ de qué otra manera podría ser? Es un humilde y limitado acercamiento al misterio de su amor, que no debe ser visto como si lo dijera todo, a primera vista, a propósito del tema. Los vocablos usados son signos que nos remiten - no sin deficiencias - a un significado que no podemos enclaustrar en palabras y conceptos. Son el camino, no la meta. Pero quienes, arriesgando sus vidas, han hecho de la opción por el pobre una práctica cotidiana - como Mons. Romero-, nos hablan de su verdadero alcance.

La preferencia significa entrar, condición ineludible, en el mundo del pobre, vivir en solidaridad con los oprimidos y marginados, rechazar la injusticia de la situación que viven, compartir su reivindicación de ser considerados personas ${ }^{15}$. Y sobre ese suelo, anunciar el evangelio a todo ser humano. La auténtica universalidad, en materia de testimonio cristiano, arranca históricamente desde esta parcialidad ${ }^{16}$. La proclamación de la buena nueva debe ir "hasta los confines de

13. Previendo ese tipo de interpretaciones, el documento "Opción preferencial por el pobre" se adelantó, afirmando que la opción por el pobre es "preferencial, pero no exclusiva" (1165, ver también 1145).

14. Es inútil recordar, además, que no hay fórmulas perfectas. No faltan quienes consideran, por ejemplo, que el término "opción" flexibiliza en exceso lo que debe ser una decisión firme. Pero, como dice muy bien M. Díaz Mateos, es "una opción no opcional, pues es verificación de nuestra identidad eclesial y cristiana" ("El grito del pobre atraviesa las nubes", en El rostro de Dios en la historia, Lima, 1996, p. 159).

15. "Optar por el pobre es entrar en el mundo de la raza, la cultura y la clase social oprimida, en el universo de sus valores, de sus categorías culturales", G. Gutiérrez "Praxis de liberación, teología y anuncio", en Concilium 96 (1974) 356.

16. Tema y término muy usados por J. Sobrino: "Medellín confiesa la divinidad y la humanidad de Cristo, pero introduce en ellas el principio de parcialidad: los pobres y la pobreza [...] La correlación primaria no es entre Jesús (y Dios) y los seres humanos en general, sino entre Jesús (y Dios) y los pobres de este mundo, a través de lo cual, 
la tierra" (Heb 1, 8). Pero comienza, según los evangelios, en la particularidad de Galilea, tierra marginada y despreciada por los habitantes de Judea, región en la cual se encuentra Jerusalén, y donde se concentra el poder religioso y político, en tiempos de Jesús. Desde el campo de los maltratados y olvidados llega el mensaje de amor universal del Dios de Jesucristo. Son aspectos inseparables.

Ellos nos hablan del amor de Dios, pauta definitiva del nuestro: "ámense como yo los he amado" (Jn 13, 34). Ese es el fundamento último del amor por toda persona y, a la vez, del compromiso prioritario con los pobres. Esto no descarta, por cierto, que haya otros motivos para esa solidaridad. Por eso, hablamos de motivo último. Como se ha dicho numerosas veces, se trata, para un cristiano, de una opción teocéntrica, centrada en el Dios anunciado por Jesús. Es decir, en su amor, en roca firme, como rezan los salmos. Pero hablar del amor de Dios, es hablar de gratuidad, como lo hace constantemente la Escritura, tema fontanal y primigenio, en la teología de la liberación.

Las formas concretas de vivir la opción preferencial por el pobre, son naturalmente variadas, según las situaciones y los procesos históricos. Deben, por lo tanto, ser examinadas y renovadas todo el tiempo. Pero, si se pierde de vista su razón final, se la mutila de un tajo y se la hace dependiente de la coyuntura, hasta el punto de no ver en ella sino la expresión de un momento histórico que, además - piensan algunos - , no correspondería más a lo que hoy vivimos en la humanidad. Fuera de la inexactitud del análisis (social, económico, cultural), implicado en tal aserción, dicha postura refleja, ante todo, una falta de percepción del sentido bíblico - por consiguiente, básico para un creyente - y teológico de la justicia y del amor de Dios.

\section{Dos lenguajes}

No hay cuestionamiento mayor al discurso sobre la fe que el que viene del sufrimiento del inocente. ¿Cómo entender a un Dios amor, en un mundo que lleva la impronta de la injusticia, del genocidio, de la violencia terrorista, del desprecio por los más elementales derechos humanos? Así de simple y de apremiante. Se trata, sin duda, de una pregunta que supera en anchura la capacidad de respuesta que tiene la teología. No obstante, es una interrogante que no podemos eludir. Sobre todo desde los países pobres y marginados. La pobreza y sus secuelas son el gran reto de nuestro tiempo. Pobreza destructora de personas, familias y naciones.

La Biblia, el libro de Job entre otros, se refiere a dos lenguajes acerca de Dios, que adquieren su pleno sentido sólo cuando se encuentran, se desafían y nutren mutuamente.

después, se podrá establecer la correlación universal", Jesucristo liberador, San Salvador, 1991, pp. 43 y 48, subrayado nuestro. 


\subsection{El lenguaje de la justicia}

La justicia es un gran tema bíblico. Con frecuencia se presenta en el binomio justicia-derecho. Hacer de él el núcleo de la vida del pueblo de Dios es una exigencia, que tiene su raíz en la voluntad de Dios. La dimensión profética de la Biblia, que desborda los libros que llevan el nombre de profetas, abunda en el recuerdo de que la fe en Dios está ligada al establecimiento de la justicia y del derecho. Más aún, sé trata de una acción que, en última instancia, tiene como sujeto a Dios mismo: "justicia y derecho sostienen tu trono", canta el salmista $(89,15)$. Ella es expresión de su santidad: Dios no es justo porque hace justicia, sino que hace justicia porque es justo.

Practicar la justicia y establecer el derecho son requerimientos que se entroncan con la santidad de Dios. Nos hablan de Dios. Para "implantar el derecho en la tierra" (Is 42,4), el Señor unge a su siervo, pone en él su espíritu (Is 42,1). Tarea que manifiesta el lenguaje profético - el dabar: acontecimiento y palabra, al mismo tiempo-, lenguaje de justicia, que nos conduce a internarnos en el amor de Dios por toda persona, en particular por los oprimidos y excluidos. La preferencia por los últimos de la historia plantea la justicia como exigencia ineludible del Dios de la Biblia, la cual va contra las desigualdades injustas, que se dan en nuestra sociedad. Decíamos, líneas más arriba, que la pobreza despoja a las personas de su condición humana y atenta contra su condición de hijas e hijos de Dios. La preferencia es un rechazo de esa situación, una pauta para establecer la justicia y el derecho, por la dignidad humana y la filiación divina de todo ser humano. Sin rechazar la pobreza (imprescindible de la opción por el pobre, como lo hemos recordado), sin compromiso con la justicia social, sin defensa de los más elementales derechos humanos no hay auténtica opción por el pobre; ni, por cierto, una preferencia que intenta corregir la inequidad social, haciendo carne en la historia la igualdad de toda persona ante Dios.

Esa es la razón por la cual la justicia de Dios y la exigencia de practicarla de parte de los creyentes, en la Biblia, siempre es presentada, en relación con el pobre. La defensa del pobre, la denuncia y el rechazo de las vejaciones que sufre, la solidaridad con su causa no son sólo expresiones de esa justicia, sino que también son su obligada verificación. De esa práctica y de esa exigencia proviene un lenguaje que nos permite hablar de Dios. Job lo descubre poco a poco, e inicia la salida de un mundo de premios y castigos, que lo encerraba en él mismo y le impedía hablar correctamente sobre Dios.

La fuente primera para el hablar sobre Dios es su autocomunicación, la buena nueva. Pero surge también -e inseparablemente- de la forma cómo, en circunstancias históricas precisas, ella es acogida. La situación de pobreza e injusticia de América Latina y del Caribe da, al hablar de Dios, acentos propios y un tono de premura, que no pueden ser eludidos. 


\subsection{El lenguaje de la gratuidad}

Esa urgencia, sin embargo, tampoco debe hacer que soslayemos la otra dimensión del hablar acerca de Dios. Nos referimos a aquella derivada del corazón mismo del mensaje bíblico: el amor gratuito de Dios, que se adelanta a nuestras obras y méritos. Objeto de contemplación y oración, ciertamente. Razón por la cual es tema preferido de la mística cristiana, tal como lo muestra la historia de la espiritualidad. Pero es también un poderoso factor de exigencia. Nada, en efecto, es más demandante que la gratuidad, la iniciativa amorosa de Dios pide una respuesta. La razón está en la frase evangélica: "dar gratis, lo que hemos recibido gratis" (Mt 10, 8).

La preferencia por el pobre no viene, en primer lugar (sería una descolocada idealización), de que sea necesariamente mejor - en un terreno moral o religioso- que los no pobres, sino debido a que se encuentra en una situación inhumana e injusta, contraria a la voluntad de Dios. El fundamento último de esa prioridad reposa en Dios, en su amor gratuito y universal. Es una cuestión de justicia, decíamos, pero no de una justicia que se refiere a normas externas, como lo es a menudo en nuestra sociedad y en el mundo religioso, sino de una justicia radical y exigente, que va al meollo de la injusticia y de la condición humana. Hablamos de gratuidad del amor de Dios, que nos ha amado primero. No, es obvio, de arbitrariedad y de capricho. La gratuidad no es dominio de lo arbitrario y superfluo. Nada más alejado al tema del amor, en el mensaje cristiano. Es cierto que, a veces en el lenguaje corriente, la gratuidad es entendida como arbitrariedad, pero este sentido está, a todas luces, excluido en cuanto hemos dicho sobre la gratuidad del amor de Dios.

Si bien es útil hablar de las dos dimensiones de la inteligencia de la fe, de los lenguajes (el profético de la justicia y el contemplativo de la gratuidad), para referirnos a Dios y comunicar el evangelio de Jesús importa repetir que ellos no pueden entenderse por separado. Si se distancian, quedarían vaciados de contenido, convirtiéndose en inauténticos y desarticulados.

El lenguaje de la gratuidad reconoce que "todo es gracia", como decía Teresa de Lissieux. El hablar profético denuncia la situación -y sus causas - de injusticia y expoliación del pobre. Sin la exigencia de la justicia, el lenguaje de la gratuidad corre peligro de ser tangencial a la historia, en la cual Dios está presente, e incluso de evadirse de ella. A su vez, el hablar de la gratuidad hace que el lenguaje de la justicia no caiga en la tentación de estrechar su visión de la historia y de Dios. Ambos lenguajes echan raíces en las condiciones de vida, en el sufrimiento y la esperanza de los insignificantes de América Latina y del Caribe y de otras áreas pobres de la humanidad. Se anudan el uno con el otro, se enriquecen recíprocamente y se hacen un solo hablar. 
Mons. Romero decía que deseaba aportar aquello que es propio de la buena nueva: la esperanza. La teología, el lenguaje sobre Dios es, finalmente, una hermenéutica de la esperanza. Un dar cuenta de los motivos que tenemos para esperar. De ello nos dio testimonio; su predicación nos convoca estos días. 